\title{
Below Knee Amputation
}

\author{
JOHS G. ANDERSEN
}

\author{
Alupe Leprosy Hospital, PO Box No. 35, Busia Market, Kenya
}

\begin{abstract}
In the management of severely damaged feet in leprosy patients amputations still play an important role. Probabilities are high that they will continue to do so for many years to come. Most leprosy patients who need that kind of surgery come under the care of medical officers with little or no training in sophisticated orthopaedic surgery. Unfortunately the traditional techniques for below knee amputation are fairly difficult to perform adequately. A less than perfect result makes it difficult to fit a prosthesis. The high risk of complications with traditional techniques, and the scarcity of prosthetic services, constitute a real need for a simple operation with a high percentage of good results.

Recently a new method has been advocated. It aims at simplifying the surgical technique, at avoiding the grave risk of ischaemic necrosis of the anterior skin flap over the cut end of the tibia, and at rendering a more suitable stump for a patellar tendon weight bearing prosthesis. The author has used this technique consistently for several years. The results have been extremely encouraging. It is suggested that its adoption as a routine method would benefit the surgeon, the prosthetist, and the patients alike.
\end{abstract}

\section{Indications}

All cases where a sound weight bearing plantar surface can not be obtained. Syme's and Pirogoff's amputations offer no real advantage. The indications for their use are largely social and psychological, particularly in communities where there are strong objections to losing any part of a limb. These operations are technically more difficult, and they yield a considerably lower percentage of good results.

\section{Contraindications}

1. Poor skin in the proximal one-third of the lower leg. Particular emphasis must be placed on the condition of the skin in the patellar tendon region, which will take most of the weight when the prosthesis is worn.

2. Chronic pre-patellar bursitis. This condition-to historically interested surgeons known as "house maid's knee"-is fairly common in communities where the women kneel down while grinding maize flour or while performing similar duties. Usually this is a relative contraindication, since careful excision of the aseptically inflamed bursa with meticulous skin suture may permit a patellar tendon weight bearing prosthesis to be fitted.

3. Infection, extending above the lower one-third of the lower leg. Again this is frequently a relative contraindication, which can be overcome with correct treatment of the original condition.

Received for publication 29 May, 1975. 
4. Degenerative oseto-arthrosis of the knee. Even if full extension of the knee is possible, such a condition will not permit a weight bearing prosthesis on an extended knee. One of two underlying conditions will usually be found. One is impairment of pain sensation around the knee and/or impairment of proprioceptive sensation of the knee structures. These conditions tend to produce a joint that is too unstable for a patellar tendon weight bearing prosthesis. A prosthesis with weight bearing thigh corset and lockable knee joint is undoubtedly the best answer. But this presupposes a standard of prosthetics that is not available to most of our patients. The other condition is intra-articular degeneration consequent on long standing contracture of the knee joint. Subluxation of the knee joint, very commonly seen in long standing contractures of the knee following post-polio quadriceps paralysis, is not a dominant feature in leprosy, but should nevertheless be definitely excluded before below knee amputation is undertaken.

Detailed radiographic evaluation of the knee joint may not be possible. Clinical assessment is normally sufficient. The following points must be fulfilled in order to ensure a below knee stump, suitable for a patellar tendon weight bearing prosthesis:

full, active extension of the knee joint,

lateral and antero-posterior stability of the knee joint,

the stump must have sufficient coverage of good quality tissue,

the length of the stump, measured as length of bone, must be at least $10 \mathrm{~cm}$

from tibial tuberosity to cut end of tibia.

A considerable proportion of our patients have a poor sense of balance. This may be due to a "psychological amputation" of an anaesthetic limb. It may also be due to deficient proprioceptive responses. It may also be due to long standing inactivity of a grossly damaged extremity or to a general despondency of the patient. This in itself is not a contraindication to amputation, but it makes the training of the patient a much more difficult and drawn out procedure.

It is worth remembering that gross infection of the foot, particularly if it involves the weight bearing surfaces, is likely to trigger off "reactions", and is also likely to be the cause of gross anaemia and protein deficiency. Much can be gained by formal amputations in spite of such obvious, general contraindications.

\section{Recommended Technique}

1. Spinal anaesthesia is recommended. Since it is unpleasant- to say the least-for anyone to listen to the saw cutting through his own bones, a fairly heavy premedication is advisable. In our experience pethidine, $100 \mathrm{mg}$, and chlorprometazine, $50 \mathrm{mg}$, given subcutaneously one half hour before arrival in the theatre is sufficient to let the patient sleep peacefully during the operation.

2. A pneumatic tourniquet is applied to the thigh. It is secured with a completely covering non-elastic bandage. After the anaesthesia has taken effect, and the required skin preparation has been completed, the leg is elevated steeply for a few minutes, and the tourniquet is quickly inflated to $\mathrm{Hg} 300 \mathrm{~mm}$.

3. Base points for the skin incisions:

(a) The selected point of division of the tibia (this should be $10-20 \mathrm{~cm}$ distal to the tibial tuberosity),

(b) A point $3 \mathrm{~cm}$ proximal to point a on the anterior border of the tibia, 


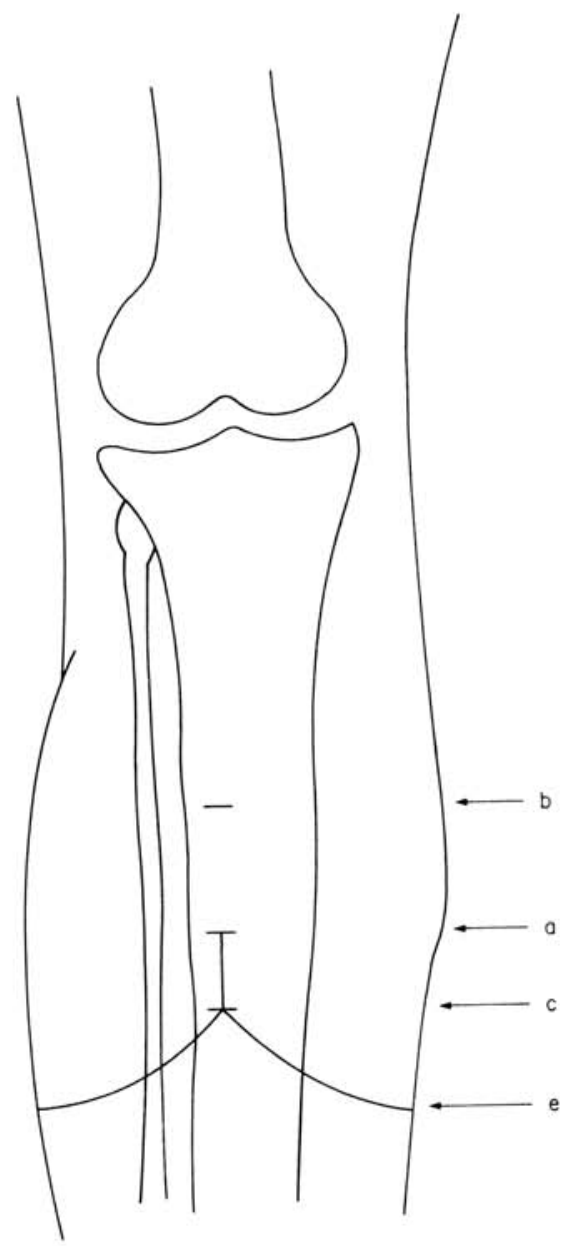

Fig. 1. Frontal view of the lower leg, showing the line of incision.

(c) A point on the anterior border of the tibia, $2 \mathrm{~cm}$ distal to point a,

(d) A point directly posterior to point a,

(e) Points in the exact mid-line of the lower leg, one on the medial one on the lateral side, both at the same height, $4 \mathrm{~cm}$ distal to points a and $\mathrm{d}$.

4. Skin incisions are carried straight through skin, subcutaneous tissue, and muscle fascia. The line of incision is from point a through $\mathrm{c}$, then in two diverging cuts through points e to point d.

5. The two semicircular flaps are raised and freed from underlying muscle for only $2 \mathrm{~cm}$. At this level the muscles are divided across. The proximal leg of the incision is carried through periosteum, which here is continuous with the muscle fascia. Major vessels are caught and ligated as they are met. Periosteum is scraped 


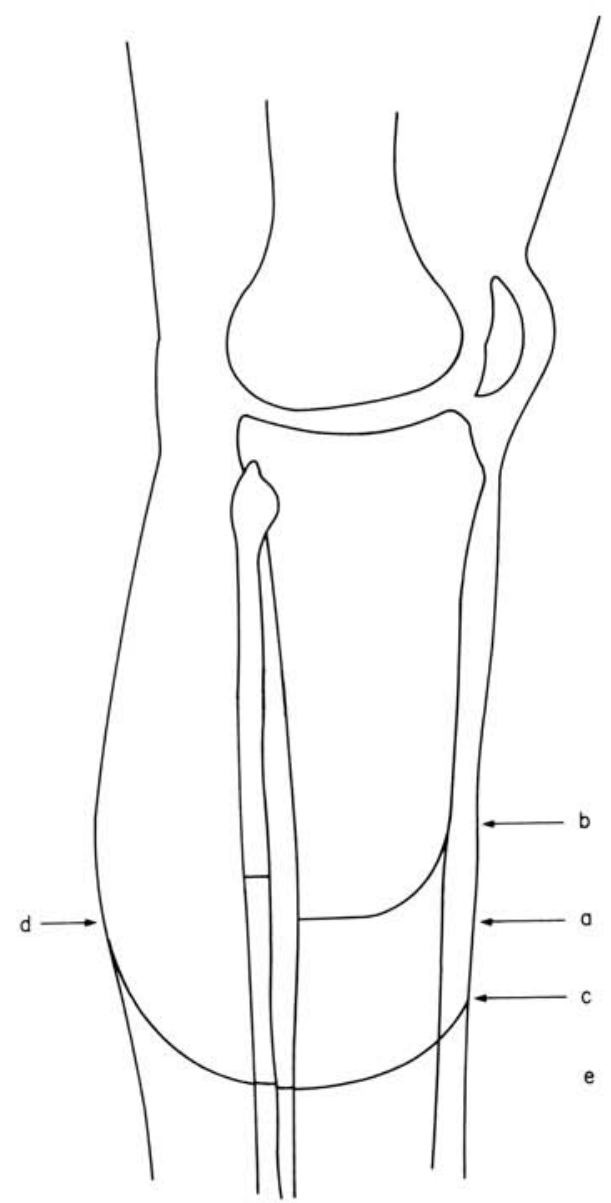

Fig. 2. Lateral view of the lower leg, showing the line of incision, and the division of tibia and fibula.

back on the tibia to point $b$. The tibia is divided at point a, after which the anterior point is cut down obliquely to minimize chances of pressure necrosis of the skin. The fibula is divided 1 to $3 \mathrm{~cm}$ above the level of the tibia cut. This ensures a cylindrical stump, which is more suitable for a patellar tendon weight bearing prosthesis than the classical, conical stump.

6. The leg is then elevated, and the tourniquet removed completely. Remaining bleeding vessels are visualized and ligated.

7. The muscle fascia is sutured in interrupted, buried knots. Any material, except silk, is acceptable, if not too heavy. No attempt is made at muscle suture. The muscles are still attached to the muscle fascia, and they fall neatly in place under the fascia suture. Skin is sutured, preferably using monofilament stainless steel wire or monofilament synthetic material. A neat skin suture is essential. If 
the skin incisions have been placed correctly, the resulting stump is cylindrical with a suture line running in the sagittal plane. Any small dog's ears should be left untouched, since their removal may jeopardize the blood supply. Anyway they invariably disappear later. If the muscles and bones have been divided correctly in relation to the skin incisions, the subfascial tissue will be under just sufficient tension to prevent the formation of dead space with the inherent danger of haematomas. Drains are therefore unnecessary. Indeed they will interfere with the neatness of the suture line, and may act as ports of entry of postoperative infections.

If the surgeon is not sure of the right level of incision, it is wiser to cut too generous flaps, both of muscle and of skin. These can always be cut back to correct size. Too short and too tight flaps on the other hand can only be corrected by cutting back the bone, which interferes with the function of the prosthesis.

8. Remaining oedema and blood is expressed, and the stump is covered with generous gauze pads, including the knee. A thin plaster of Paris shell is moulded round the stump, including the distal part of the thigh, with the knee fully extended.

9. In the ward the stump is kept elevated for 48 hours. From the third day the patient can start isometric quadriceps exercises.

10. The plaster shell is removed on the 14th day, and the patient can start active knee exercises against increasing resistance. The stump is covered in formative bandage.

11. The sutures are removed on the 21 st day. Stump bandaging continues.

12. Approximately 4 weeks after the operation the patient can usually be fitted with the first prosthesis. It is important to keep in mind that the stump will remodel and shrink while this first prosthesis is worn. A second prosthesis should not be considered a failure, but rather a necessity of the technique.

This amputation technique was originally developed for geriatric patients with ischaemic necrosis and sclerotic arteries. Under these conditions the use of a tourniquet is strictly contraindicated. Elevation of the stump postoperatively is likewise avoided. While these points do not arise from leprosy per se, nevertheless a number of our patients may fall inside the group where these precautions are indicated.

\section{References}

Persson, B. M. (1974). Sagittal incision for below-knee amputation in ischemic gangrene. $J$. Bone Jt. Surg. 56-B, 110.

Sarmiento, A., May, B. J., Sinclair, W. F., McCollough, N. C. and Williams, E. M. (1970). Lower extremity amputation. Clin. Orthop. 68, 22.

Tracy, G. D. (1966). Below-knee amputation for ischemic gangrene. Pacif. Med. Surg. 74, 251. 